\title{
Towards reliable video transmission over sparse MANETs in emergencies
}

\section{Rumo à transmissão segura de vídeo em sparse MANETs em emergências}

\begin{abstract}
Video delivery in a mobile ad-hoc network that can be deployed by members of an emergency service in an incident zone is an appealing tool for emergency and rescue services, but has not been studied yet. In order to design and test a suitable solution, we have generated realistic evaluation scenarios by modeling fireman action plans and GPS traces from real situations. We propose an overlay network solution with routing and reliability mechanisms. The Emergency Overlay Routing (EOR) protocol is a reactive protocol integrated into a store-carry-forward architecture. It selects ferry nodes to transport video data from a camera in the Incident Area to the Incident Chief's node, looking for the minimum delay, but reliable, candidate. We also propose a simple credit based mechanism (RTCP + ) to improve the communication reliability. The evaluation of the whole system shows a great improvement against previous results and

Keywords: Emergency and rescue. Sparse MANET. Routing.

Resumo: Entrega de vídeo em uma rede móbil ad-hoc em emergências é uma aplicação interessante para os serviços de emergência. Para desenhar e provar uma solução util cenários realistas foram criados a partir da modelagem dos planos de emergência e mostras GPS coletadas em situações problemas dede overlay é proposta como soluça a os Emergency Overlay Routing (EOR) é um protocolo de routing reativo integrado em uma arquitetura store-carry-forward. protocolo seleciona nodos ferry para transportar o vídeo desde a câmara na Area do Incidente ate Intervenção, buscando o retardo mínimo e a fiabilidade dos candidatos. Um mecanismo baseado em crédito é proposto para melhorar a fiabilidade da comunicação. A avaliação do sistema mostra uma grande melhoria com respeito a sistema mostra uma grande melhoria com

Palavras-chave: Emergência e resgate. MANET de pouca densidade. Routing. Transporte de Vídeo. Multimídia.
\end{abstract}

CABRERO, Sergio: G. PAÑEDA, Xabiel: PLAGEMANN Thomas; MELENDI, David; GAR'CÍA, Roberto. Towards emergencies Informática na Educação: teoria \& prática, emergencies Informatica na Educação: teoria \& pratica,

\section{Sergio Cabrero}

Universidad de Oviedo

Xabiel G. Pañeda

Universidad de Oviedo

\section{Thomas Plagemann}

University of Oslo

David Melendi

Universidad de Oviedo

Roberto García

Universidad de Oviedo

\section{Introduction}

$\mathrm{I}$

$\mathrm{n}$ the occurrence of an accident, fire or flood, emergency services are deployed aiming to protect population and goods, and, when possible, bring the crisis to an end as soon as possible. These catastrophic events may happen anywhere and unexpectedly, making it hard to establish effective preventive measures. One of the most useful tools for an emergency service in the field is to have communication among members, but it is difficult to find reliable channels. For example, emergency dedicated public networks (e.g. TETRA, VHF or WiMaX networks) can be deployed to support fire extinction in forests, although they imply high costs. However, they may be burned out or powered down under intense fire. In the case of urban 
environments, which already have communications infrastructure (e.g. cellular networks), it has been proved that these systems often become jammed by the victims, rendering them useless for the rescue teams. Packet radio devices are traditionally used for voice, because of their reliability and acceptable range. However, their capacity is not enough for data transference at a minimum rate.

Mobile ad-hoc networking is a feasible alternative to provide data sharing in such an environment. Each member of a rescue team can be equipped with a terminal (e.g. WiFi) that establishes direct communication with their teammates in close proximity and can be used to reach other colleagues further afield. From the network perspective, each device is both an end host and a router that forwards others' information. Since devices are mainly carried by people or vehicles, the network topology is dynamic. Other potential disadvantages of these networks arise from the uncertainty caused by the mobility (route changes, partitions) and the shared channel (packet losses, congestion). Nevertheless, their great advantage is their independence from an existent infrastructure and other networks. Thus, emergency services would have an instantaneous communication network, independent from others, reliable and secure, in other words, tailored to their needs. In many cases, this would mean the possibility of data transference where it was not possible before, although the communication range would normally be smaller.

Receiving video from the Incident Area(s) in the point where it is coordinated (command and control center where the Intervention Chief is located) is very appealing for the emergency services. Video would help them to foresee potential risks, analyze the situation and assign resources efficiently. Thus, emergency services can maximize the performance of the deployed resources. For example, a fireman with a camera can send video of an accident in a tunnel, allowing experts to examine the evolution of the structures without entering the area. Otherwise, this information would have to be simply an oral description of the firemen, with unavoidable flaws. Sometimes they are able to receive images using a mobile unit camera, and a helicopter as a repeater which, apart from being an expensive solution, is not always possible in field situations which are not open (tunnels, underground caves, buildings). An ad-hoc network can be used to deliver this video, although the quality and especially the delay may be affected. A few firemen deployed in the Incident Area would form a sparse network with isolated network partitions, which will cause delay in the transmission. However, in these situations, the emergency services consider it much better to have this video, even if it is delayed, that not to have it at all.

In this paper, we study video transport over sparse mobile ad-hoc networks (MANETs) deployed in an emergency situation where video recorded at the Incident Area must be delivered to the Intervention Chief. Limitations for such a system are the uncertain node movement, wireless multi-hop links instability and mobile device resources (mainly battery). In our previous work (CABRERO et al., 2009), we designed and implemented (in Java) an overlay network architecture (called MOMENTUM) that provides the store-carryforward paradigm (ZHAO et al., 2003) over the standard IP architecture. Note that storage is not a big constraint due to the great capacity of devices compared with the video transmitted (e.g. 6000 seconds of a $500 \mathrm{Kbps}, \mathrm{CIF}$, MPEG2 video $240 \mathrm{MB}$ ). Our solution considered all nodes of the MANET as part of an overlay network that can be used to store and relay video. In other words, all the nodes have MOMENTUM installed and running. In addition, video was received and delivered as RTP in the endpoint nodes, so it was possible to connect with standard servers (or cameras) and clients. Finally, the overlay network communicates with the standard network protocol OLSR, which allows cross-layer improvements, such as network topology awareness in the overlay routing decisions. The first experiments, carried out in random movement scenarios, proved store-carry- 
forward paradigm as a good alternative for video in these networks, but we detected the necessity of studying realistic scenarios to provide smarter routing decisions for the video packets. Therefore, we maintain the core philosophy from our previous work and focus in this work on more realistic scenarios and propose better routing alternatives. For that reason, we have studied fire services, their protocols and collaborated with the Asturian Fire Service (Bomberos de Asturias/112). A simplified mobility model of an emergency scenario is presented and used to analyze our solution. Then, we propose a novel overlay routing protocol tailored for emergency scenarios. The goal of this protocol is to minimize the delay of packets in a probably partitioned sparse MANET. Our hypothesis is that we can benefit from the "a priori" knowledge of these scenarios to predict future movement, thus, to find the best nodes to store the video and eventually deliver it to the Intervention Chief. In our previous work (CABRERO et al., 2011), we compared its performance with the ideas behind PROPHET, a general purpose routing protocol for delay-tolerant networks (DTNs). Since ad-hoc networks are prone to disruptions and failures that cause high packet losses, we introduce a credit mechanism to control video packet flow. Then, we carry out a new evaluation considering different credit values.

The remainder of the paper is organized as follows. Section 2 analyzes relevant related work. In Section 3, we explain our model for emergency scenarios. Our overlay architecture proposal is introduced in Section 4. The Emergency Overlay Routing protocol is described in Section 5. Section 6 provides details of the RTCP+ reliability mechanism. Section 7 exposes the evaluation of the different components. Finally, Section 8 presents conclusions and future work.

\section{Related Work}

There are several studies that can be considered relevant for the work presented in this paper. First of all, the dependence between node mobility and applications running in an ad-hoc network is an important issue. Previous studies, like (BAI et al., 2003), emphasize the relationship between the parameters of mobility and the performance of routing protocols for ad-hoc networks. Therefore, part of the research community is trying to reproduce real life scenarios into mobility models (CAMP et al., 2002). Not only do they make experimentations and repeatability easier, but also real world GPS traces are difficult to obtain. Emergency and rescue operations are not an exception and there exist proposals like (ASCHENBRUCK et al., 2007). This model, called the Disaster Area mobility model, takes into account different zones (incident site, casualty treatment area, transport zone and hospital zone) and defines the movement of units between them. We focus our research on the zone referred to as incident site, which in our opinion has not been modeled with enough detail by the state of the art. For that reason, the scenarios for this paper are built with the model presented in Section 3, which, based on our experience, defines more accurately the personnel moving in the Incident Area.

Routing in ad-hoc networks has sparked a lot of interest in recent years. When it is possible to establish multi-hop communication, because devices are in the same network partition, proactive and reactive protocols have been proposed. While proactive solutions, like OLSR (CLAUSEN et al., 2003), try to keep an updated routing table, reactive ones, like AODV, only look for a network route when it is needed. Then, their main task is to detect topology changes produced by mobility or disconnections. When the network is aimed to transport video, congestion, packet losses and other difficulties emerge, see (LINDENBERG et al., 2011) for more details. Therefore, enhanced routing solutions for video in MANETs have been given. They are mainly based on multipath (KOMPELLA et al., 2007), hierarchical, or QoS routing.

Routing in a partitioned network is a different problem, which is defined in detail by (JAIN et al., 2004). First of all, a paradigm, like store-carry-forward (ZHAO et al., 2003), is necessary to transmit packets 
between nodes in different partitions. Then, the appropriate ferry must be found. In some cases, mobility is known in advance (MEGURU et al., 2004), e.g. in space communications or networks using public transport. In that case, routing is a matter of passing messages to the right node taking available resources into account. However, mobility is unpredictable in some situations. Hence, routing protocols have to guess the best ferry to transport the information to a different partition. In this area, there are some proposals based on epidemic routing (VAHDAT et al., 2000) that spreads messages in the network whenever node buffers support it. These approaches are not adequate for video delivery, due to the large amount of packets generated by a video source. PROPHET (LINDGREN et al., 2003) represents a solid alternative in these situations. This protocol calculates a probability of encounter for every node in the network, which is updated depending on the contacts between nodes. Basically, PROPHET assumes that the node that contacts most with another has a higher probability of encountering it again in the future. A transitivity property is also considered. So if a node A contacts frequently with a node $B$, which also contacts frequently a node $\mathrm{C}$, the probability of encounter between $A$ and $C$ also increases. More details on PROPHET are given in subsequent sections.

The emergency and rescue situation may be seen, at first, like an unpredictable mobility scenario. However, there exist common behaviors of the personnel in most of the missions. Mobility could be considered somewhere between known and unknown situations exposed before. Movement is not completely free or random, but there are no previously defined movements. Consequently, we propose a protocol that considers previous contacts between nodes, but also takes into account the patterns of mobility in emergency scenarios, which other state of the art protocols do not consider.

\section{Emergency Scenarios}

The design of a new technological solution for the emergency and rescue tasks requires a good understanding of the protocols followed by emergency services and their organization. This is more crucial in the case of studying MANETs deployed by these services, because personnel movement and location are very important. This information may help us to discover repeating patterns that eventually may be used to transport video better. For this purpose, we attended emergency trials run by the regional fire department of Asturias in Spain (Bomberos de Asturias/112). We studied their plan of action (112 ASTURIAS, 2007) and examined some GPS traces from their vehicles under different situations. We also consulted other fire tactics manuals, such as (NORMAN, 2005).

The human and material resources assigned to an emergency depend on, among other factors, the existence of victims, the areas affected and the potential risks for the population (such as intoxication in chemical leaks). However, the methodology followed in them and the hierarchy assumed by the participants is very similar, just at different scales and with specific material and human resources. Next, we explain hierarchy described in the Asturian regional plan for emergencies (112 ASTURIAS, 2000). Note that plans have the same general guidelines everywhere, thus, this study could be effortlessly applied to other regions. For each area, there are one or more teams, each of them with a team leader. They are commanded by an Intervention Chief, who is in direct charge of this zone. The Intervention Chief is normally located in a safe place, some hundred meters from the Incident Area. If several zones are affected, this structure is replicated in each. Coordination between zones and among different emergency services (police, fire departments, medical service, etc.) deployed in the area is carried out in the Advanced Command Post. This post is usually located a few kilometers away from the incident areas. Finally, the Central Command Post manages all the incidents occurring in a region and it is in charge of moving and assigning resources. Figure 1 shows a diagram of the emergency 
hierarchy. Information flows from the Incident Area to the Central Command Post, while Orders and Commands go in the other direction.

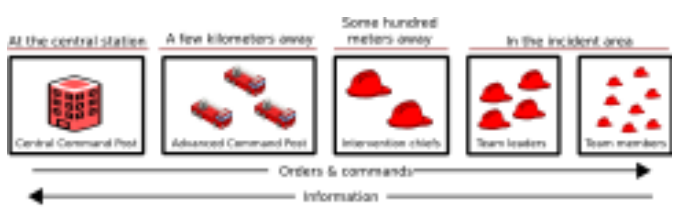

Figure 1 - Hierarchy in an emergency scenario

The hierarchy of the personnel in the emergency is intimately related to the position and mobility that they have in the scenario. In this paper, we focus on what happens between the Incident Area and the Intervention Chief, because it is where the deployment of a MANET is most meaningful. The other locations are static and may get easier access to communication infrastructures. They usually have easier access to power sources too, which is obviously relevant. Furthermore, most of the in the field decisions are taken by the Intervention Chief, thus, it is important for him/her to have as much information as possible. We will consider simple scenarios with just one Intervention Chief, one Incident Area and one or more teams dispatched to the emergency. We have observed the following patterns relevant to the MANET deployment:

- Often direct connection between the Intervention Chief and the members in the area is not possible. They may be some hundred meters apart, which is too great a distance for $\mathrm{WiFi}$ technology.

- Teams are dispatched for a mission from the point where the Intervention Chief is. Normally, they return there when the mission is finished to rest for a certain amount of time.

- There are constraints for the time that a team stays in the Incident Area. These may be externally imposed, such as the autonomy of an oxygen tank, or related to their capacity and the intensity of the work they have to accomplish.

- Normally, teams move together to and from the Incident Area. They are assigned a vehicle and use it for that purpose.

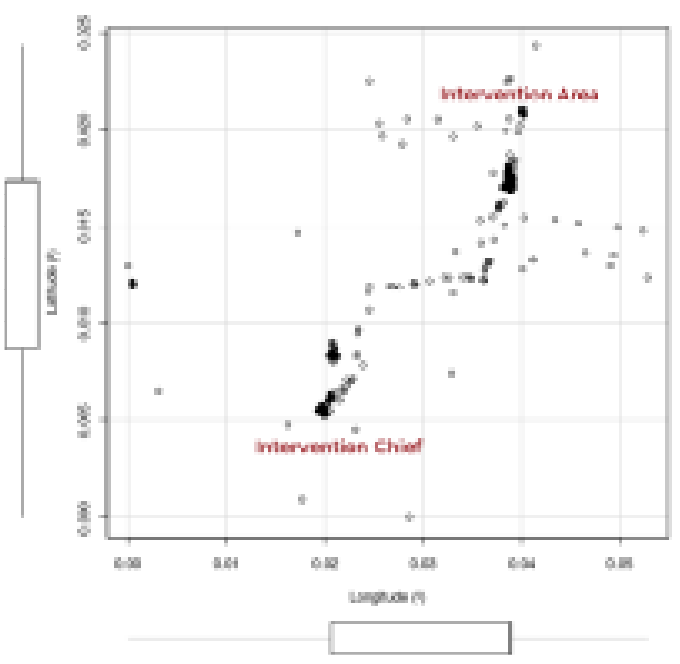

Figure 2 - Firemen GPS traces in a chemical accident trial

Figure 2 shows the GPS traces ${ }^{1}$ gathered mainly from vehicles in a chemical accident trial run by $112 /$ Bomberos de Asturias. Two different zones with people going back and forth can be clearly discerned. For this paper, we have modeled our evaluation scenarios mimicking this behavior. A rectangular zone is defined as the Incident Area and the Intervention Chief is located at a fixed position outside that zone (e.g. at a distance of 200 meters). In each scenario, there may be one or more teams. Each team has a vehicle and four firemen. Figure 3 shows how the behavior of each team is modeled. All teams are in the Intervention Chief area at the beginning of the emergency. Each team stays there for a random time (e.g. 5-10 minutes) and then moves by vehicle to the Incident Area. The vehicle is parked there and firemen start to move freely during a period of time, the Intervention Time (e.g. 10-25 minutes). Afterwards, they return to the vehicle and come back to the Intervention Chief. Each team repeats this process until the end of

${ }^{1}$ Latitude and Longitude have been modified for privacy issues 
the emergency. Finally, there is a fireman with a camera that moves randomly inside the Incident Area. Our goal is to deliver the video recorded by this node to the Intervention Chief.

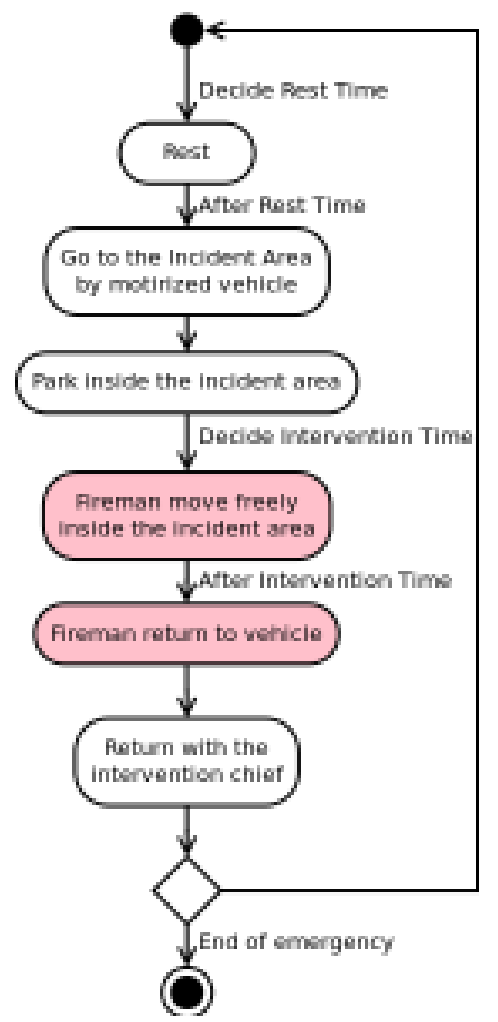

Figure 3 - Team mobility activity diagram

\section{Overlay Network}

From the previous section, it can be concluded that a MANET deployed in an emergency would often be sparse and prone to partitions. Connectivity between a camera and a video player would not be possible; we need another means of transporting the video. We propose an overlay network architecture to deliver the content produced by a camera to a video player of the Intervention Chief. This approach has the advantage of being compatible with existing protocols in both the application and the transport/network level. Our system is able to receive RTP packets from a camera and aims to deliver them to a video player. We propose a set of overlay protocols grouped in different components to cope with this transmission. In our current approach, we consider that all nodes in the network are members of the overlay, which means that they have this middleware. However, we aim for a more general scenario in the future, where there may be other nodes that are not in the overlay, but may be part of the MANET.

As we have said, a network route from the camera to the Intervention Chief's node would be an exception in these scenarios. Based on the store-carry-forward paradigm, the movement of nodes can be used to transport video packets. These are called ferry nodes and how to select them is crucial for the successful video delivery. For that purpose, we propose the Emergency Overlay Routing (EOR) protocol. Its goal is to select the best next overlay hop to reach the Intervention Chief's device. It is also well known that ad-hoc environments are prone to failures and disruptions that cause packet losses. The mechanisms designed for the RTCP+ protocol add reliability to the RTP video packets transmitted in every overlay hop. In addition, there are common mechanisms that both EOR and RTP/RTCP+ should implement, that is the goal of the Store-carry-forward Transport component, see (CABRERO et al., 2009). Although this component aims for a wider role in the system, for the experiments carried out here, it checks whether the destination for a packet is connected or not, in order to avoid packet losses and inefficient transmissions. It also limits the maximum sending rate of a node to $5 \mathrm{Mbps}$ to avoid unnecessary UDP buffer overflows. Finally, there is a Crosslayer component that aims to extract information from the OLSR network routing protocol and serve it to the other overlay components. In Figure 4, the components of this architecture are illustrated.

The expected behavior of our system is the following. First, RTP packet videos are produced in one node of the network (the camera). These packets are stored and, if the Intervention Chief's node is not connected, the EOR protocol is encouraged to find the next hop towards him/her in a DTN path (a ferry node). Once a suitable ferry has been found, RTP packets are transferred to him using RTCP+ and Storecarry-forward Transport mechanisms, all of 
them over UDP and following the network route proposed by OLSR. The receiver node will store the packets and repeat the same operation. Packets are not replicated in the network and, as will be thoroughly explained later, packets are not retransmitted. This operation is repeated until these video packets reach the Intervention Chief. Once there, the overlay will hand them out to the video player. Although it could be a standard video player, the service would be much better using an application specially designed for this environment. It is out of the scope of this paper to explain in detail its features, but since video chunks may arrive disordered, it should be able to store them and make them available to users as they arrive.

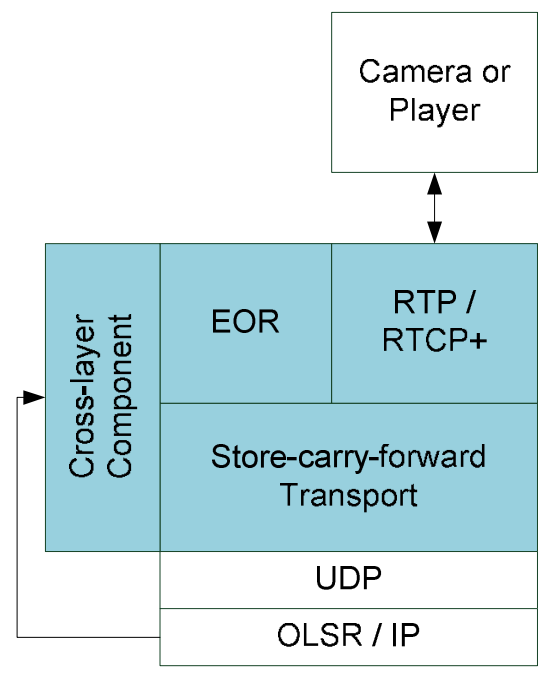

Figure 4 - Overlay Network architecture

In the following sections, we will introduce the novel components of this architecture: the Emergency Routing Protocol and RTCP+.

\section{Emergency Overlay Routing}

One of the goals of our overlay architecture is to deliver the video produced by the camera with the minimum delay possible and ensuring an acceptable quality. When the video player can not be reached using a network route, the Emergency Overlay Routing (EOR) can contribute by finding the best ferry nodes. It looks for the minimum delay option taking into account route reliability. Every node which has stored video packets, because it has generated or received them, must check whether any other node in the same network partition is a better ferry towards the Incident Chief. Note that the protocol can be generalized for any other video sink in the network. To make efficient overlay routing decisions, it should be beneficial to consider the "a priori" knowledge acquired from the study of emergency scenarios. This should be a big advantage over other general purpose routing protocols used in DTNs. In addition, topology information, like existent routes and neighbors, is extracted from the network routing protocol OLSR. Finally, remember that all nodes in the network are considered members of the overlay; otherwise some of the protocol mechanisms must be modified.

Bearing this in mind, we have designed an overlay routing protocol tailored for emergencies. It relies on two basic mechanisms. On the one hand, the ferry, or next overlay hop, selection uses information gathered from different nodes in the network partition and also from the local node. This function chooses the best ferry from the nodes in the partition. Note that the node performing the selection may not find a ferry better than itself, and then it would keep the packets. On the other hand, there is the protocol itself, which is used by a node to exchange the information needed for the ferry selection.

\subsection{EOR Ferry Selection}

The goal of this function is to identify the best ferry in a network partition. We consider the following parameters as the most important to consider in such decision.

The type of unit carrying the network device. There may be different types of units that move at different speeds or with known movement patterns. In general, a vehicle is more reliable than a fireman for several reasons, i.e. less battery constraints, less probability of breaking the network device during the intervention, or more likely to go back to the Intervention Chief. In addition, they are often parked in the Incident Area, which may imply more 
stable links. The type of unit can be hardcoded in the devices.

The time passed since the last meeting with the Intervention Chief. For example, if we assume similar intervention times for all the teams, those team members that arrived at the Incident Area first will be the first ones to meet the Intervention Chief. Note that PROPHET would consider that the last arriving will be the first to meet the destination. This parameter can be obtained from the routing table of OLSR. A timestamp is saved when a network route to the Intervention Chief's node exists in OLSR routing table.

The number of network hops to reach the ferry node. The longer the network route is, the higher the risk of losing packets due to collisions in the multihop transmission. This is even more problematic if the information sent is not a single packet, but many of them. This parameter can also be obtained from the OLSR crosslayer communication.

In this paper, we combine them in the following equation to establish the value of a node as ferry. The higher this value is, the better the ferry.

\section{Ferry value $=$ seconds since last encounter-(node type)/hops}

\subsection{EOR Protocol}

The Emergency Overlay Routing protocol (EOR) is responsible for collecting the information needed for ferry selection. It is running on top of OSLR and resembles to a large extent AODV. It is reactive, i.e., only if a node needs to forward video data it looks for the best ferry in the given partition. If the network partition has been stable since it previously forwarded video data it simply uses the same ferry as before. Otherwise it broadcasts an EOR Route Request (EORReq), which is answered with EOR Route Responses (EORRes). To do this efficiently EOR uses the nodes selected as Multipoint Relays (MPRs) by OLSR. Using a sequence number for each packet in the overlay network, already broadcasted messages are not sent again, avoiding network loops. It is important that all the nodes are members of the overlay, which means that they are able to perform this retransmission. Otherwise, the route request mechanism should be redesigned. EORReq contains the node type and the seconds passed since the last encounter of the node with the Intervention Chief. Therefore, a node receiving such a message is able to calculate the ferry values of both the requester and itself. Hence, EORRes are sent only if a node considers itself a better ferry than the node asking for the route.

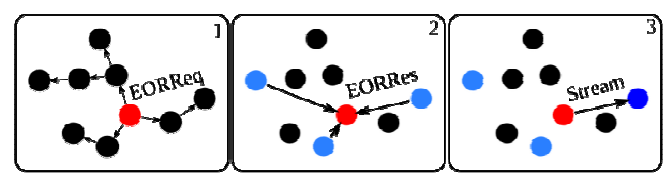

Figure 5 - Overlay routing protocol overview

Figure 5 shows the message exchange of EOR. First, a node sends an EORReq (1). These messages are processed by the nodes as shown in the activity diagram of Figure 6. All ERRes received by the overlay route requester (2) are considered to perform ferry calculation. The finally step (3) represents the ferry receiving the stored video stream.

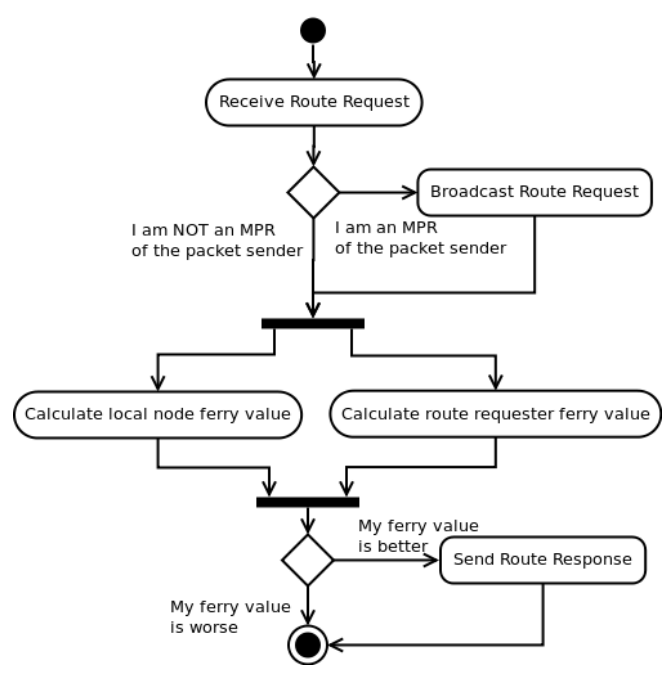

Figure 6 - EORReq receiver activity diagram

There is an exception for this behavior: when the destination of the overlay route (i.e. the Intervention Chief) is detected in the partition, it is always selected as next 
hop. Figure 7 summarizes this in a state diagram for EOR.

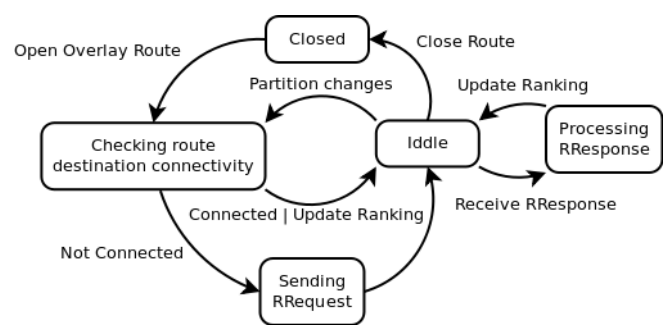

Figure 7 - EOR protocol state diagram

\section{RTP / RTCP+}

Our previous evaluations, which we will summarize later, show that most of the packet losses are caused by temporary disruptions, for example due to ARP or OLSR malfunction. The vision of the MANET offered by the protocols is different to the reality and most packets sent while this situation persists are dropped. For example, if a node moves out of reach of another while they are exchanging packets, all of them will be lost until OLSR is aware of the disconnection. The longer (hop-wise) the route is, the stronger this effect is, because route network reconfigurations take some time to propagate in OLSR. Similarly, ARP resolutions that are slowly or wrongly solved (e.g. because a reply is lost) produce incoherence in the information given by OLSR and ARP, which ends in packet drops at lower levels. Thus, many packet losses are not individual packets, but bursts of them that are dropped during network protocols malfunction.

We have designed a credit based protocol to ease their effect in the video transmission between overlay nodes. It is a simple mechanism that controls RTP packet flow by limiting the number of packets that can be transmitted without being acknowledged by the receiver. We call it RTCP+. The goal is not to carry out retransmissions, but to check if the network route is working appropriately. For that reason, acknowledges are not done per packet, but per group of packets and retransmissions are not considered. The core idea is to limit the maximum number of packets that could be lost.
The overall behavior of the protocol is described by its state machine in Figure 8. When a node in the overlay network has video packets that must be delivered to the Intervention Chief, it requests a next overlay hop (a ferry if we are not in the Intervention Chief partition) to the EOR protocol. Once a suitable node is found, it is asked for credit (i.e. the maximum number of packets the receiver is willing to receive). When the credit is received, the node starts sending video packets until the credit is over. The receiver must send new credit once half of the expected packets are received, so waiting times are avoided if the communication is reliable. If no credit is received before all packets are sent, a new credit request is sent. This process goes on until there are no packets left, no credit is received for 1 second (timeout value) or changes are detected in the network topology. This last occurrence may lead to selecting a different ferry, so the EOR is requested again.

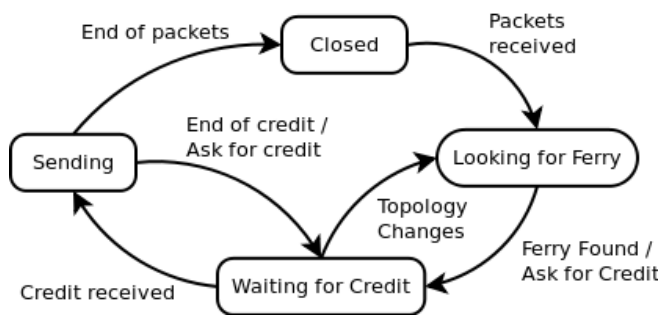

Figure 8 - RTP / RTCP+ protocol state diagram

Figure 9 represents all the messages exchanged between two nodes involved in video transmission. The receiver receives an EOR Request that is answered because it is a good ferry to the Intervention Chief. The sender receives the EOR Responses and selects the receiver as the best option to store these video packets in the partition. Thus, the RTP/RTCP+ component begins the transmission sending a Credit Request that is answered with a W credit message. Then, $\mathrm{W}$ packets are marked for sending to the receiver. Once $\mathrm{W} / 2$ packets have been received, $\mathrm{W} / 2$ additional credit is allowed to the sender. Packet losses, changes of the topology and the end of the stored packets in the sender may finish this cycle. It is important to note that for simplicity, packets 
are removed in the sender once they are sent, so a packet lost in the network is impossible to recover. Retransmissions may be part of the future work.



Figure 9 - EOR with RTCP+ protocol sequence diagram

\section{Evaluation}

In this section the evaluation of our protocols is described. First, we describe the evaluation framework and the scenarios used. Then, we summarize the results of our previous evaluation of EOR, published in (CABRERO et al., 2011). Finally, just two scenarios are chosen to evaluate the improvements that RTCP+ makes to video delivery using different credit values.

\subsection{Evaluation framework}

Real time simulation over ns $-3^{2}$ has been used to make the experiments. Each ns-3 network node is connected to a virtual machine ${ }^{3}$ through virtual network interfaces (taps). Each virtual machine runs MOMENTUM, which is implemented in Java, and an OLSR daemon, apart from the monitoring processes (tcpdump, sar) or the video server (VLC) in the camera node. The configuration of OLSR is the default provided in its RFC (CLAUSEN et al., 2003). The

\footnotetext{
${ }^{2}$ http://www.nsnam.org

${ }^{3}$ http://lxc.sourceforge.net/
}

stream sent by VLC and captured by MOMENTUM in the camera node is a version of the Coastguard sequence taken from the Video Trace Library ${ }^{4}$. It has been encoded in MPEG-2 at $500 \mathrm{Kbps}$ and repeated in a loop until the end of the scenario. All of this is run on a single machine with enough resources to carry out the simulations.

Table 1 - Experiments summary

\begin{tabular}{|c|c|c|}
\hline Scenario Type & Connected & Sparse \\
\hline Incident Area & $200 \times 200 \mathrm{~m} 2$ & $1000 \times 1000 \mathrm{~m} 2$ \\
\hline $\begin{array}{c}\text { Distance to } \\
\text { the Incident } \\
\text { Area }\end{array}$ & $100 \mathrm{~m}$ & $400 \mathrm{~m}$ \\
\hline Teams & 2 & 4 \\
\hline $\begin{array}{c}\text { Intervention } \\
\text { Time }\end{array}$ & $\mathrm{U}[300,1500] \mathrm{s}$ \\
\hline Rest Time & $\mathrm{U}[300,600] \mathrm{s}$ \\
\hline Node Range & \multicolumn{2}{|c|}{$\sim 100 \mathrm{~m}$} \\
\hline Duration & \multicolumn{2}{|c|}{$6000 \mathrm{~s}$} \\
\hline Seed & 1 to 5 & 6 to 10 \\
\hline Runs & \multicolumn{2}{|c|}{3 to 5 } \\
\hline
\end{tabular}

The ns-3 scenarios reproduce the mobility described in Section 3. Each MANET node has a device with the DSSS $11 \mathrm{Mbps}$ version of $802.11 \mathrm{~b}$. The Friss propagation model is used for losses and the Constant Speed propagation model for delay. To obtain realistic communication ranges the parameter RxGain of the WiFi physical layer was set to -16 , which means a range of slightly larger than 100 meters. There is one node for the Intervention Chief and another for the camera. There are also nodes for the firemen and the vehicles. These are grouped in teams of 4 firemen and 1 vehicle. Table 1 describes the two types of scenarios considered: Connected and Sparse. In the Connected one, there may be network routes between the camera and the Intervention Chief. Two teams are deployed adding up to a total of 12 nodes. On the contrary, a network route between the camera and the Intervention Chief is nearly impossible in a Sparse scenario. There are 4 teams, thus, 22 nodes. Times of Intervention and Rest were calculated as a uniform random variable of 5 to 25 minutes and 5 to 10 minutes respectively. The total duration of the scenario was 100 minutes.

${ }^{4}$ http://trace.eas.asu.edu/ 
Each type of scenario (Connected and Sparse) was repeated using 5 different seeds for the random numbers selection. This generates 5 different mobility patterns and different time choices for nodes and teams. The Connected scenarios were generated using 1 to 5 as seeds. 6 to 10 were used for the Sparse ones. Each of them is repeated 3 times for each routing protocol.

\subsection{EOR vS. PROPHET}

This section summarizes the results obtained in the comparison of EOR and PROPHET carried out for (CABRERO et al., 2011). The goal of this evaluation was to measure the benefits of the Emergency Overlay Routing Protocol in the store-carryforward architecture of MOMEMTUM. For that reason, possible reliability or congestion control mechanisms have not been included in the architecture. This means that RTCP+ was not included. In these experiments, the values assigned to each type or node are: 0 for the camera, 1 for the firemen, 2 for the vehicles and 3 for the Incident Chief. Two metrics are considered important: the amount of video packets delivered and their total delay. Both can be easily measured end-to-end using traffic traces. This should provide enough information to compare the two overlay routing protocols. The following subsection explains how it has been integrated in MOMENTUM for that purpose.

\subsubsection{PROPHET}

We compare the Emergency Overlay Routing protocol with PROPHET to analyze the benefits of EOR against other proposals. We have selected PROPHET because it is a well-known protocol in the DTN community. Furthermore, PROPHET proposes almost an opposite philosophy for selecting ferries. It is a widely accepted probabilistic protocol for DTNs, which has been drafted by IETF DTNs Research Group. They have also implemented PROPHET over their DTN architecture, but we did not find it suitable for our purposes. To make a fair comparison, using the same store-carryforward mechanisms, we have implemented the next hop selection of PROPHET inside our own architecture. Instead of using the ferry calculation from EOR, MOMENTUM uses the encounter probability defined in PROPHET. The higher the probability is, the better the ferry. The other protocol mechanisms of the Overlay Routing Protocol (Route Requests and Responses) remain the same.

The encounter probability considers past encounters to predict future node connections. So each time the node (e.g. the Intervention Chief) is in the partition the encounter probability for this node is updated as:

$$
\mathbf{P}=\mathbf{P} \_ \text {old }+\left(\text { 1-P_old ) } \mathbf{P} \_0\right.
$$

Then, at each moment the probability is calculated:

\section{$\mathbf{P}=\mathbf{P} \_$old $\cdot \mathbf{Y}^{\wedge}$ (time since last $\mathbf{P}$ calculation)}

$P$ is the current probability of encounter with the given node (in our case the Incident Chief). $\quad P_{-} 0$ is a constant representing the initial probability. P_old is the value of last probability of encounter calculated. $\mathrm{Y}$ (gamma) is the aging constant that represents how much time passed since last $P$ calculation affects the new $P$. Values of gamma and the initial probability have been taken from the values recommended in (LINDGREN et al., 2003).

$$
\begin{gathered}
\text { P_o }=0.75 \\
\mathbf{Y}=0.98
\end{gathered}
$$

Finally, for simplicity we have not implemented the transitivity properties of PROPHET. We keep this in mind for future work, but since the Emergency Overlay Routing protocol is also suitable for adding transitivity and it is not considered in this version; we believe this is a fair comparison.

\subsubsection{Results}

Figure 10 summarizes the results of the previously mentioned experiments. It shows the Experimental Cumulative Distribution Function (ECDF) of all the video packets in all the scenarios. They have been classified in four groups, depending on the overlay routing protocol and the type of scenario, so each line gathers information of 5 variations of the same scenario (using different seeds for the random number generation) and 3 
runs for each variation. The delay of lost packets, i.e. packets that did not reach the Intervention Chief, is considered as infinite. Therefore, the figure represents delay and packet losses showing that EOR outperforms PROPHET in these scenarios when the two metrics are considered. Using the same transport protocols and architecture (MOMENTUM), our overlay routing solution is more likely to deliver a packet and to do it with a lower delay. The second important outcome is that the rate of packets delivered is extremely low (below 10\%). It is even lower in the Sparse scenarios, with delivery rates around $1 \%$.

In connected scenarios, when nodes are able to communicate easily with a potential ferry (vehicles), communication is more reliable and results of EOR are better. On the contrary, in sparse environments, it is more difficult for both protocols to have potential ferries and, therefore, to deliver the packets. In addition, the camera node has fewer contacts with vehicles and more overlay hops may be necessary to reach the Intervention Chief; for example, from camera to fireman, and from fireman to vehicle (or to another fireman). This means higher risk of packet losses too.

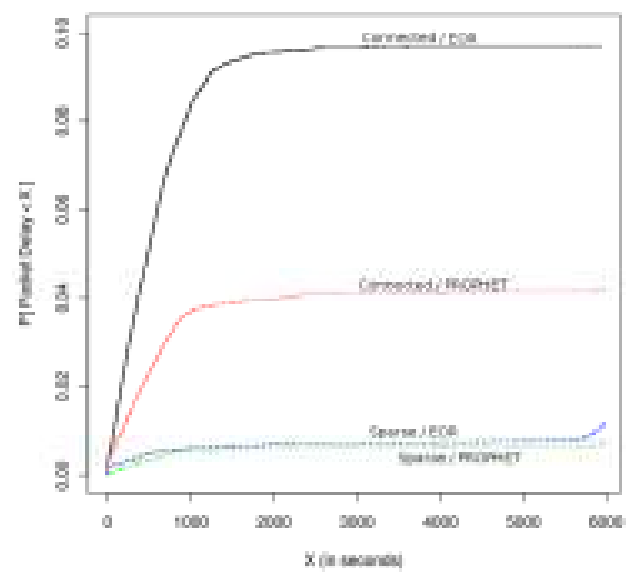

Figure 10 - Video packets delay ECDF

Ten different mobility scenarios have been evaluated, five of them Connected (seeds 1 to 5 ) and five of them Sparse (seeds 6 to 10). Figure 11 shows the total number of packets delivered and packets from the 3 runs have been added. In the connected cases EOR is clearly superior, while in the Sparse scenarios there are several different situations. Observe that delivery is very low, thus it is difficult to extract relevant conclusions.

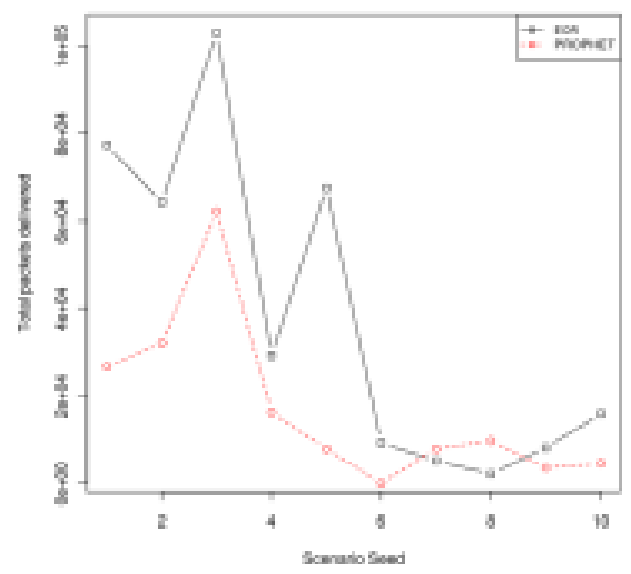

Figure 11 - Video packets delivery in each scenario

A more detailed analysis of the results reveals the reasons for the higher reliability and lower delay of EOR in the Connected situation and the not so good performance in the Sparse one. The former Incident Area size eases communication between the Camera node and the others in the area, including vehicle nodes. Considering that the ferry calculation favors vehicles against other nodes (they have a higher node type value), EOR tends to select them as ferries. In the $200 \times 200$ meters Incident Area, the Camera may find a 1-hop or 2-hop transmission with a vehicle easily. Since they are parked, routes are more reliable. On the contrary, PROPHET ferry selection eventually turns out in less reliable transmissions, because vehicles are considered at the same level as other nodes. In the Sparse Incident Area it is more difficult to find these reliable transmissions between the Camera and vehicles. Both protocols send a lot of packets through transmissions between nodes more likely to move. Furthermore, there are more nodes (22) so large network routes are more frequent. Therefore, packet losses are higher than in the Connected case.

The overall performance of the system is still far from a final solution. The percentage 
of packets eventually delivered to the client is very low. Packets are not discarded by MOMENTUM, therefore, the explanation for this is in the lower layers and the lack of connectivity.

In many sparse scenarios, nodes connect infrequently. Therefore, sometimes it is just not possible for the camera to find a suitable ferry for every video packet generated. The delivery rate metric we have considered takes into account all the packets produced by the server, which includes those that the camera node was not able to forward in the duration of the scenario simulation. Sometimes packets are not lost, but never sent by the camera. Many others are buffered by intermediate nodes. Note that these packets may also be useful for an offline analysis.

Packet collisions are common in wireless ad-hoc networks and RTS/CTS mechanism does not always perform as expected (XU et al., 2003). Moreover, MOMEMTUM nodes send packets continuously that will compete among themselves over multi-hop routes. This is also aggravated if several nodes try to send packets to the same node (e.g. the best ferry in the partition or the video destination), which is a likely situation.

The information provided by the network routing protocol (OLSR) is not real time. This protocol carries out periodic queries in the network to discover neighborhood and routes. If a route changes or a node disconnects, it is not immediately detected. Of course, lower times for discovery broadcasts could be configured, but assuming the higher network consumption by OLSR. This impact has been analyzed by others (VOORHAEN et al., 2006). Finally, independence of ARP and the routing protocol also contributes to losses. Packets sent during ARP resolutions are normally lost (CARTER et al., 2003).

\subsection{RTCP+}

We have evaluated our overlay network solution in the Connected (seed 3) and Sparse (seed 10) scenarios with best results from the EOR evaluation. The goal is to analyze the improvement of RTCP+ in packet delivery, but also its overhead. We have considered different credit values from 10 to 120 packets and a special case with an Infinite credit. Due to the results of previous experiments, we have also improved the prototype implementation, making it more efficient and faster in the decision taking. Finally, we have modified the values of the node type parameter of EOR. Now they are: 0 for the camera, 10 for the firemen, 20 for the vehicles and 30 for the camera. We pay special attention to the video delivery rates, the packet losses and the overhead caused by EOR and RTCP+.

\subsubsection{Connected scenario}

The connected scenario chosen for the evaluation of RTCP+ was the one with seed 3 . We show the average results of 5 runs for each credit value $(10,30,60$ and 120) and for an infinite credit value. Figure 12 summarizes the results obtained for each case. The delivered packet rate is the number of packets received by the client divided by the total number of packets produced by the camera. The buffered packet rate is the number of packets that are stored in the nodes of the overlay, but have not reached the Incident Chief, again divided by the packets produced by the camera. Finally, any other packet that has not been delivered or is not buffered in any node at the end of the experiment is considered as lost.

There is a clear influence of the credit value in the percentage of lost packets. This is reasonable, since the more credit a receiver gives, the more packets can be lost in case of network disruption. However, this lost rate affects more the buffered packets, while the delivered packets are almost constant for all the finite credit values. The case of infinite credit is similar to the results obtained for the previous EOR evaluation with less than $10 \%$ of the packets delivered and most of them lost. Thus, the simple mechanisms proposed by RTCP+ greatly improved the reliability.

There are two main causes why buffered packets are not delivered. Either the nodes carrying them have not found the Incident Chief or an appropriate ferry node before the end of the scenario. In this case, EOR 
should be improved to select better ferries. However, it could be that these encounters existed, but the transference was not fast enough to pass all the stored packets. Then, it is the duty of RTCP+ to optimize this transference. In this case, it would also be important to ensure that the most important packets for the user QoE (Quality of Experience) are delivered first.

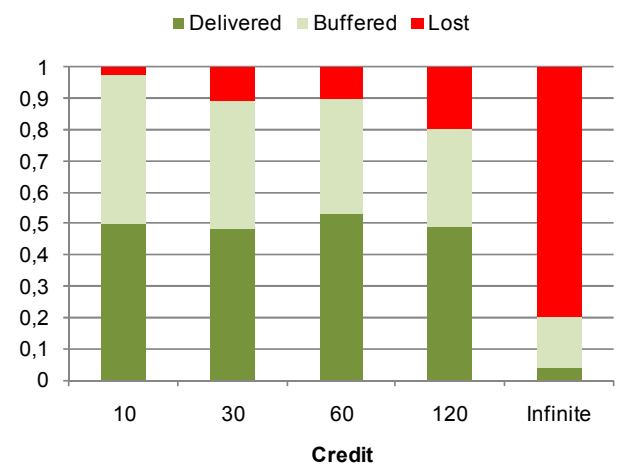

Figure 12 - Connected Scenario delivery summary

Another crucial point is the overhead produced by EOR and RTCP+ to transport the video. It is important to keep it low, since resources are very scarce in an ad-hoc network. We have counted the number of packets produced by each protocol in all nodes and by the video source and represented them in Figure 13. It is always below $30 \%$, which at first sight may appear a large number. However, we have to take into account the size of these packets too. While a video packet size is of several hundred kilobytes, the size of an EOR or $\mathrm{RTCP}+$ message is below $1 \mathrm{~KB}$. Therefore, the overhead is not significant. If we consider the performance of the different credit values, we can not appreciate big differences. The smaller credit value (10) is more sensitive to packet losses. For that reason, RTCP+ will be restarted more than for other values, producing slightly more RTCP+ messages.

In an ad-hoc network packets are retransmitted several times until they reach their destination. Thus, it is also important to consider this overhead too. We have measured the number of bytes of each protocol transmitted by each node in the
MANET. Figure 14 shows the average of adding the bytes sent by all the nodes in all the runs for each credit value. We can see that, in the worst case, non-video packets are less than $1.5 \%$ of all the bytes sent by a node. Therefore, we can conclude that overhead of EOR and RTCP+ is insignificant against the video traffic.

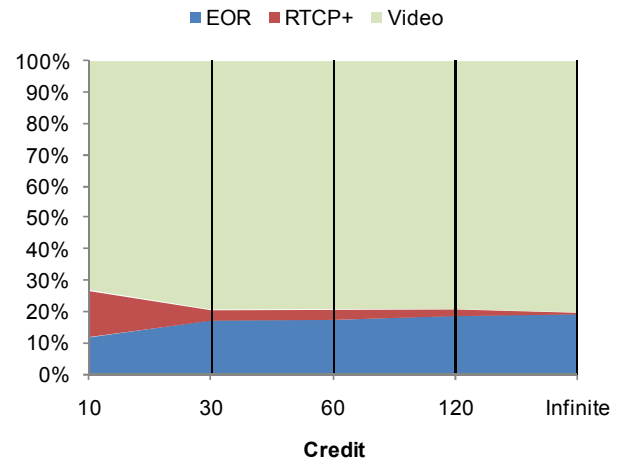

Figure 13 - Average packets sent by each protocol

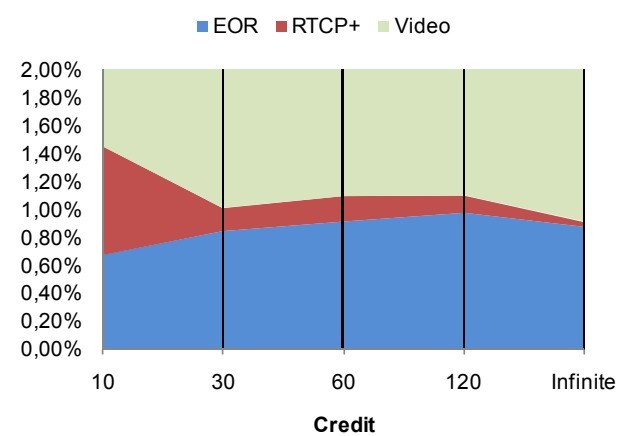

Figure 14 - Bytes sent to the network by protocol

\subsubsection{Sparse scenario}

We have carried out an analogue experiment with the sparse scenario with seed 10. Figure 15 shows the delivered, buffered and lost packet rates. What is noteworthy is that for a credit value of 10 , the packet delivered rate is a $20 \%$ lower than for any of the other values, except Infinite. As we have mentioned, a small credit value is more sensitive to packet losses. If 5 packets are lost with a credit value of 10 , the EOR/RTCP+ process (Figure 9) has to be restarted, producing a delay and a bigger overhead. In the sparse 
scenario, it is more difficult to connect with potential ferries, thus, the opportunity of forwarding packets must not be wasted. For that reason, the connected scenario is not so sensitive to this effect. Another interesting result is that packet losses are lower in the sparse scenario than in the connected one. It is not simple to explain this effect, since the scenarios are completely different, but this may be due to the network partition size. While in the sparse scenario partitions are smaller, in the connected scenarios it is more possible to find bigger partitions where more nodes compete for the network resources.

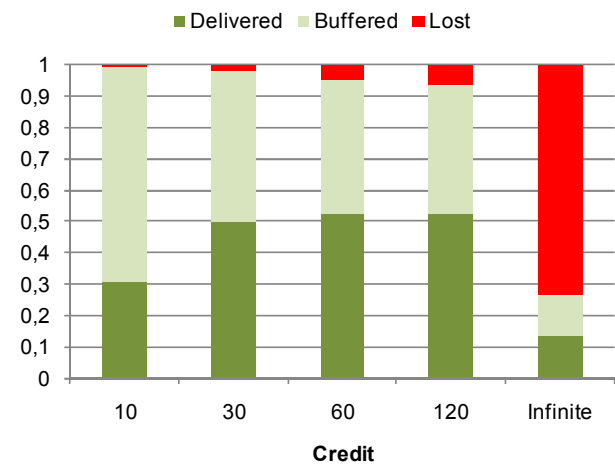

Figure 15 - Sparse Scenario (seed 3) delivery summary

We have also measured the overhead. The most noticeable result is that the packets produced by EOR are less than in the connected scenario. The reason is that EOR broadcast requests are triggered by topology changes. In a bigger area, these changes are less frequent. Due to the high sensitivity of the 10 credit value, its overhead is much higher than any of the others.

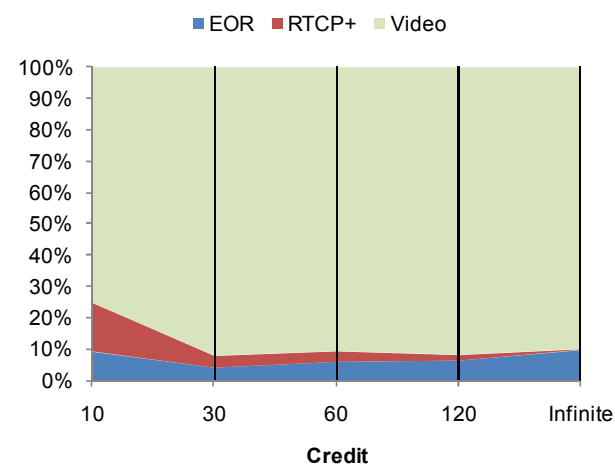

Figure 16 - Average packets sent by each protocol

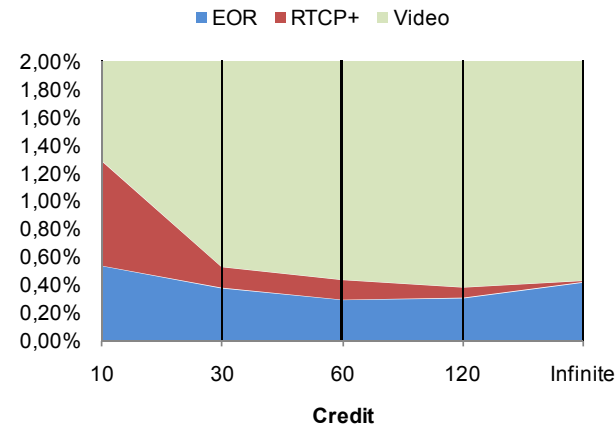

Figure 17 - Bytes sent to the network by protocol

\section{Conclusions and Future Work}

In this paper, we have presented a novel architecture to distribute video in emergency scenarios. We have studied emergency services, proposing realistic evaluation scenarios. We have designed an overlay network solution with two main components: EOR protocol and RTCP+. These cope with ferry selection and reliability of the communications respectively. Previous evaluations of EOR showed the weaknesses of the system against packet losses in the ad-hoc network. However, a simple credit mechanism has shown great improvement of the overall system. Furthermore, the overhead introduced is insignificant compared with the video traffic.

Small credit values lead to lower packet losses, but they do not necessarily produce more packets delivered to the Incident Chief. In the connected scenarios 10 seems to be the best option. However, in the 
sparse, 30 seems better. Due to the heterogeneity of emergency scenarios, establishing a credit value is not an easy task. Other approaches must be taken, such as dynamic values depending on the context. This would probably be true for many other mechanisms and protocols. Thus, part of the future work should be oriented to develop context aware mechanisms in the overlay network. Another goal should be to deliver more packets whenever possible, but taking into account that in partitioned MANETs there is an upper boundary imposed by the mobility of the nodes in the scenario. Finally, it is important that delivered packets are the most relevant to the user and that the system only assumes the loss of less relevant packets. For that reason, future work should also consider QoE.

\section{Acknowledgements}

This work has been funded by the Spanish National Research Program (FUTURMEDIA Project TSI2007-60474), the Norwegian Research Council (DT-STREAM Project 183312/S10), and it received support from the EU Network-of-Excellence CONTENT.

\section{References}

112 ASTURIAS. Plan Territorial de Protección Civil del Principado de Asturias (PLATERPA). Government of the Principality of Asturias, 2000.

ASCHENBRUCK, N.; GERHARDS-PADILLA, E.; GERHARZ, M.; FRANK, M.; MARTINI, P. Modelling Mobility in Disaster Area Scenarios. Proc. 10th ACM-IEEE Int. Symposium on Modeling, Analysis and Simulation of Wireless and Mobile Systems (MSWIM), pp. 4-12, 2007.

BAI, F.; SADAGOPAN, N.; HELMY, A. The IMPORTANT Framework for Analyzing the Impact of Mobility on Performance of Routing for Ad Hoc Networks. AdHoc Networks Journal - Elsevier Science, Vol. 1, Issue 4, pp. 383-403, November 2003.

CABRERO, S.; G. PAÑEDA, X., PLAGEMANN, T., GOEBEL, V. and SIEKKINEN, M. Overlay solution for multimedia data over sparse MANETs. In Proceedings of the 2009 International Conference on Wireless Communications and Mobile Computing: Connecting the World Wirelessly, ser. IWCMC '09. New York, NY, USA: ACM, 2009, pp. 1056-1061.

CABReRo S.; G. PAÑEdA, X.; PlAgemanN, T.; MELENDI, D.; GARCíA, R. An Overlay Routing Protocol for Video over sparse MANETs in Emergencies. VI Congresso Ibero-americano de Telemática. Mayo 2011.

CAMP, T.; BOLENG, J.; DAVIES, V. A survey of mobility models for ad hoc network research. Wireless Communications \& Mobile Computing (WCMC): Special issue on Mobile Ad Hoc Networking: Research, Trends and Applications, vol. 2, no. 5, pp. 483-502, 2002.

CARTER, C., YI, S., KRAVETS, R. ARP considered harmful: manycast transactions in ad hoc networks. In: Wireless Communications and Networking (WCNC). Vol. 3, IEEE pp. 1801-1806, 2003.

CLAUSEN, T.; JACKET, P. Optimized Link State Routing Protocol (OLSR). IETF RFC 3626, 2003.

JAIN, S., FALL, K., PATRA, R. Routing in a delay tolerant network. SIGCOMM Comput. Commun. Rev. 34 145-158, 2004.

KOMPELLA, S.; MAO, S.; HOU, T., SHERALI, H.D. Cross-Layer Optimized Multipath Routing for Video Communications in Wireless Networks. IEEE Journal on Selected Areas in Communications, 2007.

LINDEBERG, M., KRISTIANSEN, S., PLAGEMANN, T., GOEBEL, V. Challenges and techniques for video streaming over mobile ad hoc networks. Multimedia Systems 17, 51-82, 2011. 
LINDGREN, A.; DORIA, A.; SCHEL, O. Probabilistic routing in intermittently connected networks. SIGMOBILE Mob. Comput. Commun. Rev. 7, 3 (July 2003), 19-20, 2003.

MEGURU, S.; AMMAR, M.; ZEGURA, E. Routing in space and time in networks with predictable mobility. Georgia Inst. Technol., Atlanta, GA, Tech. Rep. GIT-CC-04-7, 2004.

NORMAN, J. 2005. Fire Officer's handbook of tactics. Fire Engineering Books \& Videos; 3 edition (May 1, 2005).

VAHDAT, A.; BECKER, D. Epidemic routing for partially connected ad hoc networks. Technical Report, Duke University, 2000.

VOORHAEN, M.; BLONDIA, C. Analyzing the impact of neighbor sensing on the performance of the olsr protocol. In Proceedings of 4th Intl. Symposium on Modeling and Optimization in Mobile, Ad Hoc, and Wireless Networks (WiOpt06), April 2006.

XU, K.; GERLA, M.; BAE, S. Effectiveness of RTS/CTS handshake in IEEE 802.11 based ad hoc networks. Ad Hoc Network Journal, 1(1), July 2003.

ZHAO, Z.; AMMAR; M. Message ferrying: proactive routing in highly-partitioned wireless ad hoc networks. 9th IEEE Workshop on Future Trends of Distributed Computing Systems, 2003.

Recebido em maio de 2011

Aprovado para publicação em junho de 2011

Sergio Cabrero

Universidad de Oviedo, Oviedo - Spain. E-mail: cabrerosergio@uniovi.es

Xabiel García Pañeda

Universidad de Oviedo, Oviedo - Spain. E-mail: xabiel@uniovi.es

Thomas Plagemann

University of Oslo, Oslo - Norway. E-mail: plagemann@uio.no

David Melendi

Universidad de Oviedo, Oviedo - Spain. E-mail: melendi@uniovi.es

Roberto García

Universidad de Oviedo, Oviedo - Spain. E-mail: garciaroberto@uniovi.es 